\title{
Aktivierende und geschlechtergerechte Arbeitsmarktpolitik
}

\author{
- den Anforderungen des Art. 3 Abs. 2 und 3 GG ist erst noch Rechnung zu tragen -
}

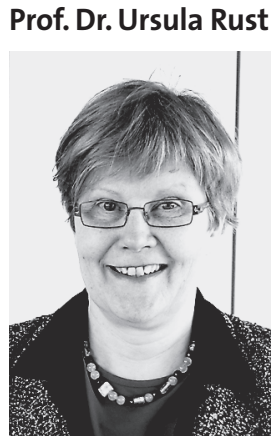

Mitglied der Kommission Recht der sozialen Sicherheit, Familienlastenausgleich des djb; Universität Bremen
Kurzfassung: Einkünfte der Niedrig- oder Nichtverdienenden werden bei Langzeitarbeitslosigkeit für die Mitglieder der Bedarfsgemeinschaft des SGB II zusammengerechnet. Überwiegend Männer werden mit der rechtlichen Konstruktion des \ 9 Abs. 2 Satz 3 SGB II zu „fiktiv Hilfebedürftigen“. Umgekehrt sind es überwiegend Frauen, wenn sie mit besser verdienenden Partnern zusammenleben, die aus den Eingliederungsleistungen des SGB II „herausgerechnet“ werden. Die Grundsicherung für Langzeitarbeitslose wird als Ort genutzt, um auf das Problem ausstehender oder mangels Unterhaltsfähigkeit nicht bestehender Unterhaltsansprüche von Kindern mit einer sozialrechtlichen Inpflichtnahme zu reagieren. Zur institutionellen Umsetzung der Gleichstellungsziele des SGB II fehlt die Einrichtung Beauftragter für Chancengleichheit für das SGB II, wie sie bis 2005 für die Arbeitslosenhilfe im SGB III vorgesehen war.

\section{SGB II-Leistungen statt Arbeitslosenhilfe}

Mit den vier „Hartz-Gesetzen“ ist 2002/2003 die aktivierende Arbeitsmarktpolitik vollständig eingeführt worden. Der Einstieg hatte schon $2001 \mathrm{im}$ SGB III - Arbeitsförderung - mit dem Job-AQTIV-Gesetz ${ }^{1}$ und neuen Elementen wie der Eingliederungsvereinbarung begonnen. Das SGB III wurde mit den Hartz-Gesetzen grundlegend reformiert. Das SGB II - Grundsicherung für Arbeitssuchende - ist für Langzeitarbeitslose anzuwenden, also nach einem Jahr Arbeitslosigkeit. Das SGB II und das SGB XII - Sozialhilfe - sind 2005 neu eingeführt worden. Das SGB XII regelt die Grundsicherung bei Bedürftigkeit und voller Erwerbsminderung oder aber der Regelaltersgrenze von 65/67.

Die Kommission Recht der sozialen Sicherung, Familienlastenausgleich des djb hat die Einführung der aktivierenden Arbeitsmarktpolitik begleitet. 2002/2003 war in der Phase des Einstiegs gleichstellungspolitische Expertise seitens der Bundesregierung nicht angefragt. ${ }^{2}$ Zur Verabschiedung der gemeinsam beratenen Hartz-Gesetze III und IV hat der djb als Korrektiv jedenfalls eine Mindestquote bei aktiven Arbeitsförderungsmaßnahmen für solche Arbeitslose gefordert, die allein aufgrund fehlender Bedürftigkeit kein Arbeitslosengeld II erhal- ten. Der djb hat außerdem die im Entwurf vorgesehene Eingliederungsvereinbarung für Dritte und damit die Möglichkeit, dass der männliche Haushaltsvorstand künftig eine Eingliederungsvereinbarung für seine Partnerin abschließen könnte, kritisiert. Das geltende Gesetz lässt einen solchen Vertrag zu Recht so nicht zu. Kritisiert wurden außerdem die Kürzungen bei den Berufsrückkehrerinnen und die Abschaffung der Beitragsfreiheit während der Pflege von Angehörigen. Der djb wies außerdem darauf hin, dass mit der gesetzlichen Einführung der Bedarfsgemeinschaft des SGB II absehbar in Ostdeutschland besonders viele Arbeitslosenhilfeempfängerinnen wegen der Anrechnung des Partnereinkommens künftig keine Leistungen mehr erhalten werden. ${ }^{3}$

Das SGB II wird seit 2005 angewendet. Es nennt als Aufgabe und Ziel der Grundsicherung für langzeitarbeitslose Arbeitssuchende, die Gleichstellung von Frauen und Männern durchgängig zu verfolgen ( $\mathbb{} 1$ Abs. 1 Satz 2 SGB II). Fraglich ist, wie dies im Rahmen einer Bedarfsgemeinschaft im Sinne des SGB II tatsächlich erfolgt oder für Grundsicherungsträger möglich ist und ob das Gleichstellungsziel ohne eine Individualisierung der Leistungen überhaupt erreicht werden kann. Auf diese Fragen konnte im September 2006 die Vorsitzende der Kommission soziale Sicherung Christel Riedel in einem Gespräch im Bundesministerium für Familie, Senioren, Frauen und Jugend aufmerksam machen. Dem djb war bereits aus der Arbeitslosenhilfe und der Sozialhilfe nach dem BSHG bewusst, wie negativ sich die Berücksichtigung von Partnereinkommen für Frauen auswirken kann. In der djb-Stellungnahme im Verfahren vor dem Bundesverfassungsge-

1 www.djb.de/Kommissionen/kommission-recht-dersozialen-sicherung-familienlastenausgleich/pm-68 und www.djb.de/Kommissionen/kommission-rechtder-sozialen-sicherung-familienlastenausgleich/sn-66 zum Job-AOTIV-Gesetz für die öffentliche Anhörung des Ausschusses für Arbeit und Sozialordnung am 10.10.2001 mit Pressemitteilung 01-13.

2 www.djb.de/Kommissionen/kommission-recht-dersozialen-sicherung-familienlastenausgleich/sn-94, pm 93, 96, 99 mit sn-98 „Offener Brief djb, dfr, BAG: Arbeitslose Frauen werden erste Verliererinnen der Wahl“ vom 6.11.2002.

3 www.djb.de/Kommissionen/kommission-recht-dersozialen-sicherung-familienlastenausgleich /pm-140/. 
richt aus dem Jahr 1992 zur damaligen Arbeitslosenhilfe 4 hieß es:

"Solche Regelungen sind geeignet, entweder aus der überforderten Familiennotgemeinschaft eine Lügengemeinschaft zu machen oder gar den Familienverband durch Überforderung zu zerstören“ ... „Ein Rechtszustand, der nur den formalen oder tatsächlichen Bruch einer persönlichen Beziehung voraussetzt, damit Leistungen ungeschmälert erbracht werden, belohnt entweder ein unsolidarisches Verhalten oder er beutet freiwillige Solidarität unter Menschen aus."

Ob die Kritik des djb, die seinerzeit für die einkommensabhängige Arbeitslosenhilfe ergangen war, auch für die Bedarfsgemeinschaft des SGB II berechtigt ist, wurde Grundlage des Forschungsprojekts „Individualisierung von Leistungen des SGB II unter Berücksichtigung von Unterhaltspflichten“. Das Projekt fand 2008 statt und wurde vom Bundesministerium für Familie, Senioren, Frauen und Jugend gefördert. Antragsteller war der djb. Durchgeführt worden ist das Projekt an der Universität Bremen - interdisziplinär in Zusammenarbeit von Rechts- und Sozialwissenschaften.

Die rechtswissenschaftliche Recherche übernahm Prof. Dr. Ursula Rust vom Bremer Institut für deutsches, europäisches und internationales Gender-, Arbeits- und Sozialrecht am juristischen Fachbereich der Universität Bremen mit Mohamad El-Ghazi, Eliane Hütter und Kathrin Schlote. ${ }^{5}$ Für die sozialwissenschaftliche Analyse war Prof. Dr. Sigrid Betzelt - während des Projekts noch Mitarbeiterin beim Zentrum für Sozialpolitik an der Universität Bremen - beteiligt mit Manuela Schwarzkopf und Tanja Schmidt.

\section{Die drei Fragestellungen zum SGB II-Projekt des djb}

Die erste Fragestellung des Projekts war, ob mit dem Bezugspunkt der Bedarfsgemeinschaft des SGB II die Gleichstellung von Frauen und Männern rechtlich und tatsächlich als durchgängiges Prinzip verfolgt werden kann und familienspezifische Lebensverhältnisse erwerbsfähiger Hilfebedürftiger berücksichtigt werden können. Die zweite Fragestellung des Projekts bestand darin, ob arbeitslosen Personen, die individuell dem Grunde nach Anspruch auf Leistungen zur Sicherung des Lebensunterhalts haben, diese aber mangels finanzieller Bedürftigkeit nicht erhalten, aktive Arbeitsförderungsmaßnahmen zugänglich sind und diese auch genutzt werden. Dritte Fragestellung des Projekts war, ob es mit dem gleichstellungspolitischen Ziel des SGB II übereinstimmen kann, zur institutionellen Seite der Gleichstellungspolitik im SGB II - anders als zum SGB III - die Institution der Beauftragten für Chancengleichheit nicht verbindlich vorzusehen.

Zum Projekt haben 2008 zwei Fachtagungen stattgefunden. Die erste war die rechtswissenschaftliche Fachtagung in Bremen am 30. Mai 2008. ${ }^{6}$ Die zweite war der sozialwissenschaftliche Workshop in Loccum vom 29. bis 30. September 2008, der in Zusammenarbeit mit Dr. Joachim Lange von der Evangelischen Akademie stattfand. ${ }^{7}$

\section{Identifizierung rechtlicher Probleme}

Die rechtswissenschaftliche Fachtagung befasste sich mit der bedürftigkeitsgeprüften Grundsicherung für Arbeitssuchende und den zugehörigen Mitgliedern der Bedarfsgemeinschaft, deren Bedürftigkeit mit der Horizontalmethode festgestellt wird. Die Tagung untergliederte sich in fünf Themenbereiche:

- Familiennot-, Einsatz- oder (fingierte) Bedarfsgemeinschaft?

- Unterhaltsrecht und Bedarfsgemeinschaft - Widersprüche und Parallelen

- Methoden der Einkommensanrechnung in SGB II und SGB XII -Verfassungsrechtliche Bedenken?

- Arbeitsförderung nach SGB II und III für Mitglieder der Bedarfsgemeinschaft - eine rechtliche oder auch eine reale Möglichkeit?

- Verfahrensrecht, Bedarfsgemeinschaft, Individualanspruch - Scheitert die Bedarfsgemeinschaft am Verwaltungsverfahrensrecht?

Die Themenbereiche waren in drei Diskussionsrunden zusammengefasst, moderiert von Dr. Christine Fuchsloch, Richterin am Landessozialgericht Berlin-Brandenburg, Prof. Dr. Kirsten Scheiwe, Universität Hildesheim und Prof. Dr. Margarete Schuler-Harms, Universität der Bundeswehr, Hamburg, alle Mitglieder des Projektbeirats und der djbKommission Recht der sozialen Sicherung, Familienlastenausgleich.

Für die Tagung wurde mit Beispielrechnungen, die für das Projekt von Mohamad El-Ghazi erstellt wurden und auf der djb-Homepage grafisch dargestellt sind ${ }^{8}$, verdeutlicht, wie bei insgesamt identischen Ausgaben unterschiedliche Auswirkungen mit der für das SGB II verwendeten Horizontalmethode im Unterschied zu der für das SGB XII verwendeten vertikalen Berechnung erzielt werden. Dem Beispiel ist der bis Mai 2009 geltende Regelsatz des SGB II zugrunde gelegt. Hinzugerechnet werden die Kosten der Unterkunft (KdU). Das Kindergeld mindert lediglich den Bedarf des Kindes und ist daher vorab abzuziehen. Die für das SGB II nicht angewendete Vertikalmethode des SGB XII ${ }^{9}$ bewirkt folgende Verteilung:

4 Einkommensanrechung bei der damaligen Arbeitslosenhilfe nach $\S$ 138 Abs. 1 Nr. 2, Abs. 3 Nr. 9 AFG nur bei nicht dauernd getrennt lebenden Ehegatten war mit Art. 3 Abs. 1 GG unvereinbar gemäß Urteil des BVerfG vom 17.11.1992 - 1 BvL 8/87, BVerfGE 87, $234 \mathrm{ff}$.

5 Siehe auch djbZ 4/2008, 194-196.

6 El-Ghazi/Hütter/Schlote, Tagungsbericht, SGb 2008, 688; Betzelt/ Rust, ZeS Report (13) 1/2008, S. 20; Betzelt/Schwarzkopf/Rust, ZeS Report (13) 2/2008, 9-11.

7 Betzelt/Lange/Rust (Hrsg.), Wer wird „aktiviert“ - und warum (nicht)? Erste Erkenntnisse zur Realisierung der gleichstellungspolitischen Ziele des SGB II, Loccumer Protokolle 79/08.

8 www.djb.de/publikationen/zeitschrift/djbZ-2009-3/djbZ-20093 f8/

9 Brühl/Schoch, in LPK-SGB II, § 9 Rn. 42 ff.; Gerlach, ZfF 2007, S. 121. 


\begin{tabular}{|c|c|c|c|}
\hline & Mutter & Vater & $\begin{array}{l}\text { 15-jähriges } \\
\text { Kind }\end{array}$ \\
\hline Regelsatz & 312 Euro & 312 Euro & 278 Euro \\
\hline KdU & 175 Euro & 175 Euro & 175 Euro \\
\hline Einzelbedarf & 487 Euro & 487 Euro & 453 Euro \\
\hline - Einkommen & - 0 Euro & - 600 Euro & - 154 Euro \\
\hline Überhang & - & 113 Euro & - \\
\hline Ungedeckter Bedarf & 487 Euro & - & 299 Euro \\
\hline Überhanganrechnung ${ }^{10}$ & - 70 Euro & - & - 43 Euro \\
\hline Individualanspruch & 417 Euro & 0 Euro ${ }^{11}$ & 256 Euro \\
\hline
\end{tabular}

Für dieselbe Konstellation und bei insgesamt identischem Ausgabevolumen wird mit der Horizontalmethode das Einkommen von 600 Euro auf alle Mitglieder der Bedarfsgemeinschaft verhältnismäßig verteilt und damit gesplittet. Die für das SGB II verwendete Horizontalmethode ergibt: ${ }^{12}$

\begin{tabular}{|c|c|c|c|}
\hline & Mutter & Vater & $\begin{array}{l}\text { 15-jähriges } \\
\text { Kind }\end{array}$ \\
\hline Regelsatz & 312 Euro & 312 Euro & 278 Euro \\
\hline KdU & 175 Euro & 175 Euro & 175 Euro \\
\hline - Kindeseinkommen & & & - 154 Euro $^{13}$ \\
\hline Einzelbedarf & 487 Euro & 487 Euro & 299 Euro \\
\hline Einkommen & 0 Euro & 600 Euro & 0 Euro \\
\hline $\begin{array}{l}\text { Einkommens- } \\
\text { anrechnung }\end{array}$ & - 230 Euro & - 230 Euro & - 140 Euro \\
\hline Individualanspruch & 257 Euro $^{14}$ & 257 Euro & 158 Euro \\
\hline
\end{tabular}

ohne Vorabzug

des KG: 230 Euro 230 Euro 214 Euro

Die Individualansprüche werden ausgehend von der Horizontalmethode anteilig reduziert. Gleichzeitig wird mit dem Splitting auch der Verdiener nach $\mathbb{S} 9$ Abs. 2 Satz 3 SGB II fiktiv hilfebedürftig, wenn der Verdienst nicht hoch genug ist, um die gesamte Bedarfsgemeinschaft aus dem Hilfebedarf des SGB II „auszulösen“. Die fingierte Hilfebedürftigkeit des Erwerbstätigen sieht das BSG als verfassungsgemäß an, da die Vorschrift dem sonst Nichthilfebedürftigen lediglich einen zusätzlichen Anspruch gewährt und ihn insoweit nicht belaste. ${ }^{15}$ Die Auswirkungen der Horizontalmethode als für das SGB II gewählte Berechnungsmethode wurde während der Tagung ausführlich diskutiert.

Für die rechtswissenschaftliche Fachtagung wurde außerdem die Diskussion über das Problem der sozialrechtlichen Inanspruchnahme ohne unterhaltsrechtliche Grundlagen vorbereitet. Die sozialrechtlichen Einstandspflichten innerhalb der Bedarfsgemeinschaft, die unter anderem für Partnerkinder im SGB II angeordnet sind, haben nur teilweise eine Entsprechung im Unterhaltsrecht. Dieses „Phänomen“ war zwar auch im Arbeitslosenhilfe- und im Sozialhilferecht bekannt. Die Inkongruenz zwischen dem Sozialrecht und dem Unterhaltsrecht ist mit dem SGB II stetig ausgeweitet worden. Für das Projekt wurden von Mohamad El-Ghazi, Eliane Hütter und Kathrin Schlote zwei Schaubilder erstellt, die mit farbigen
Pfeilen für verheiratete Eltern und für Patchworkfamilien das Auseinanderfallen zwischen Unterhaltsrecht und sozialrechtlichen Einstandpflichten verdeutlichen und auf der djb-Homepage eingestellt sind. In Rechtsprechung, Literatur und in den Medien findet bisher die sogenannte „Stiefkinderproblematik" besondere Beachtung. In dieser Konstellation ist das Problem der Inkongruenz von Sozial- und Unterhaltsrecht offenkundig. Nachdem in der Instanzrechtsprechung erhebliche Zweifel an der Verfassungsmäßigkeit des $\mathbb{S}$ Abs. 2 Satz 2 SGB II geäußert wurden, hat das BSG die verfassungsrechtlichen Zweifel verworfen. ${ }^{16}$ Prof. Dr. Peter Derleder, Universität Bremen, untersuchte in seinem Referat folgende Konstellationen auf die Existenz einer Bedarfsgemeinschaft bzw. eines zivilrechtlichen Unterhaltsanspruchs:

1. Die Konstellation vor der Bildung einer Bedarfsgemeinschaft.

2. Die Bildung einer Bedarfsgemeinschaft mit einem hilfebedürftigen und einem nicht hilfebedürftigen Partner.

3. Der Auszug aus dem Hotel Mama.

4. Der Solidarbeitrag der Stiefeltern.

5. Die temporäre Bedarfsgemeinschaft.

6. Der Rückzug in die Ursprungsfamilie.

Für die rechtswissenschaftliche Fachtagung wurde vom Projekt außerdem eine Übersicht zum Zugang zu aktivierenden Leistungen verteilt. ${ }^{17}$ Schon seit dem Hartz III-Gesetz ist der Zugang zur Weiterbildung für Arbeitssuchende damit geöffnet, dass die bisher für die berufliche Weiterbildung des SGB III erforderliche Vorbeschäftigungszeit gestrichen wurde und für die Ermessensleistung „nur“ Arbeitslosigkeit im Sinne des SGB III vorliegen muss. Hartz IV hat mit der „Durchstiegsnorm“ des $\mathbb{} 16$ Abs. 1 SGB II außerdem für Langzeitarbeitslose mit SGB II-Bezug den Zugang zu ausgewählten Aktivierungsmaßnahmen des SGB III geöffnet. Die Förderinstrumente sind Ende 2008 mit einem im Verhältnis zum bisherigen Katalog größeren Entscheidungsspielraum für die Grundsicherungsträger zusammengefasst worden.

Inwieweit die Aktivierung zumutbar ist, ist im SGB II geregelt. Nach $\mathbb{1 0}$ Abs. 1 Nr. 3 SGB II sind Arbeitssuchende von der Verpflichtung zur Erwerbsarbeit in der Regel ausgenom-

10 Die Überhanganrechnung kann nach verschiedenen Methoden erfolgen (Verhältnis-, Kaskaden- oder Kopfteilmethode); an dieser Stelle erfolgt die Berechnung anhand der Verhältnismethode. Dabei wird der Überhang im Verhältnis des Einzelbedarfs zum ungedeckten Bedarf angerechnet.

11 Somit wäre der Ehemann Nichtleistungsempfänger.

12 Nach Mecke, in Eicher/Spellbrink, § 9 Rn. 9; LSG Hessen, BeckRS 2007, 44445, Schwabe, FPR 2007, S. 359 (361); Spellbrink, NZS 2007, S. 121 (122).

13 Da das Kindeseinkommen nicht auf den Bedarf der Eltern angerechnet werden darf, muss dieses vorher abgezogen werden.

14 Rundung nach $\S 41$ Abs. 2 SGB II; dies kann bei verschiedenen Berechnungsmethoden zu minimalen Abweichungen führen.

15 BSG, Urt. v. 7.11.2006, B 7b AS 08/06 R; a.A. Schoch in Rothkegel, Sozialhilferecht, S. 310 Rn. 16; Brühl, LPK-SGB II, § 9 Rn. 24 und 32.

16 Urteil v. 13.11.2008 - B 14 AS 2/O8 R, SGb 2009, 32-33.

17 www.bigas.uni-bremen.de/. 
men, wenn sie Betreuungspflichten für Kinder unter drei Jahren haben. Anschließend ist jede „körperlich, geistig oder seelisch" mögliche Arbeit zumutbar, soweit kein weiterer Ausschlussgrund i.S.v. $\$ 10$ Abs. 1 Nr.1-5 SGB II vorliegt. Prof. Dr. Karl-Jürgen Bieback, Universität Hamburg, wies in seinem Referat ausgehend von den Zumutbarkeitsregeln darauf hin, dass es im SGB II einen Konflikt zwischen Erwerbsbürgerorientierung und dem traditionellen Familienmodell dahingehend gebe, dass zwar das SGB II grundsätzlich arbeitsmarktorientiert sei, gleichzeitig aber eine Verfestigung ungleicher familiärer Verteilungsstrukturen zu befürchten sei.

Prof. Dr. Corinna Grühn von der Hochschule Bremen leitete ihr Referat zu der provokanten Frage „Scheitert die Bedarfsgemeinschaft am Verwaltungsverfahrensrecht? “, das mit Eindrücken und Erfahrungen aus der Praxis von Dr. Lothar Schneider vom Landessozialgericht Baden-Württemberg ergänzt wurde, mit einem Zitat aus einer BSG-Entscheidung ein:

„Andererseits wäre es nicht unangebracht, das Konstrukt der Bedarfsgemeinschaft, das das SGB XII nicht kennt, in seiner bisherigen Ausprägung (insbesondere $\mathbb{9}$ II 3 SGB II) ersatzlos zu streichen. Die erörterten und geschilderten rechtlichen und tatsächlichen Probleme belegen, dass die Einführung der Bedarfsgemeinschaft keineswegs der Praktikabilität dienlich ist." 18

Dann arbeitete die Referentin die grundsätzliche Problematik der Mischkonstruktion der Bedarfsgemeinschaft im Verfahrensrecht heraus. Der Beitrag wurde nach der Tagung mit dem Titel „Verfahrensrecht, Bedarfsgemeinschaft und Individualanspruch nach dem SGB II“ in: SGb 2008, 513515 veröffentlicht.

\section{Zur Praxis der Aktivierungsmaßnahmen}

In der sozialwissenschaftlichen Tagung wurden die Erkenntnisse der rechtswissenschaftlichen Tagung als Stellschrauben für eine geschlechtergerechte Ausgestaltung der Grundsicherung einleitend zusammengefasst. Als Ergebnis der ersten Tagung, bei der mehrfach fehlende Daten für eine fundierte sozialwissenschaftliche Forschung als Problem benannt worden waren, hatte außerdem Kathrin Schlote den Beitrag zu den rechtlichen Grundlagen der Statistik im SGB II vorbereitet. Dr. Betzelt leitete die sozialwissenschaftliche Diskussion mit einem Überblick zu den Grundsatzfragen ein.

Für die erste Fragestellung des Projekts war die Erkenntnis der sozialwissenschaftlichen Forschung über die Auswirkungen der verstärkten Partnereinkommensanrechnung im SGB II für Alleinerziehende grundlegend. Ein Teil der Betroffenen vermeidet die Gründung neuer Beziehungen zu Dritten außerhalb der Bedarfgemeinschaft oder sieht zumindest davon ab, mit dem neuen Partner einen gemeinsamen Haushalt zu begründen. Es kam zu Fällen, in denen der gemeinsame Haushalt mit einem Partner verlassen worden ist. Einige Männer fragen sogar bei Beratungsstellen an, ob sie sich das Zusammenziehen mit der neuen Partnerin, die Kinder hat, „überhaupt leisten können“.
Zu der rechtlichen Konstruktion des $₫ 9$ Abs. 2 Satz 3 SGB II, dass Partner - und dies sind wegen unterschiedlicher Einkommenssituation eher selten Frauen - einer erwerbsfähigen und im Sinne des SGB II hilfebedürftigen Person zu „fiktiv Hilfebedürftigen “ werden und für sie somit sozialrechtliche Ansprüche und Mitwirkungspflichten des SGB II zur Anwendung kommen können, wird in der sozialwissenschaftlichen Forschung als Folge benannt, dass der Partner dies nur mit dem Verlassen der gemeinsamen Wohnung verhindern kann, eine aus der Arbeitslosenhilfe als verfassungsrechtlich problematisch bekannte Konstellation.

Geschlechtsspezifische Verteilungswirkungen des SGB II beim Zugang zu Leistungen, Fragen der Chancengleichheit beim Zugang zu Beratung, Vermittlung und Eingliederungsleistungen sowie die Interaktion von „Kundin oder Kunde Vermittlerin oder Vermittler“ im Beratungs- und Vermittlungsprozess als Einflussgrößen für den Zugang zur Arbeitsförderung waren die Themenblöcke, zu denen die vorliegende sozialwissenschaftliche Forschung präsentiert wurde. Vorgestellt wurde außerdem von Tanja Schmidt ihre im Rahmen des SGB II-Projekts erstellte Untersuchung über Arbeitslose, die keine Leistungen beziehen. Hierzu lagen bisher keine Daten vor. Ausgewertet wurden dazu Angaben aus dem sozio-ökonomischen Panel.

Der letzte Block der Tagung behandelte die institutionelle Verankerung der Gleichstellungspolitik im SGB II. Eindeutiges Ergebnis war, dass das Gleichstellungsziel des Gesetzes die institutionelle Begleitung erfordert. Von Teilnehmenden wurde darauf hingewiesen, es solle unbedingt versucht werden, die anstehende Reform des SGB II in diesem Sinne zu nutzen.

\section{Fazit}

Die interdisziplinäre Untersuchung hat strukturelle Probleme erkannt, die mit dem Konstrukt der Bedarfsgemeinschaft im SGB II verbunden sind und sowohl eine gleichstellungs- als auch familienpolitische Dimension haben. Der Gesamtbericht des SGB-II-Projekts mit über 250 Seiten liegt dem Ministerium vor. Die Veröffentlichung wird derzeit vorbereitet. ${ }^{19}$

Die Erkenntnisse des SGB II-Projekts können der djb-Kommission wichtige Grundlagen geben, um die rechtspolitischen Forderungen für eine geschlechtergerechte Grundsicherung konzeptioneller als bisher in die anstehende Veränderung des SGB II einbringen zu können. Hier ist zu hoffen, dass anders als bisher, der djb zukünftig seitens der Fachministerien beteiligt werden wird.

Eine Erkenntnis für die weitere Diskussion im djb ist, dass während im Steuerrecht für Ehepaare mit überdurchschnittlichem Verdienst das Splittingverfahren eine erhebliche steuerliche Entlastung bewirkt, ${ }^{20}$ im Sozialrecht für Bedarfsgemein-

18 BSG-Entscheidung vom 7.11.2006, Az. B 7b AS 8/06 R, SGb 2007, 308314.

19 Die Dokumentation der sozialwissenschaftlichen Fachtagung ist erschienen und kann als Loccumer Protokoll 79/08 bei www.loccum.de/protokoll/shop/shop.html bestellt werden.

20 Siehe dazu Maurer und Spangenberg in diesem Heft. 
schaften mit unterdurchschnittlichem Einkommen eine Art von „sozialrechtlichem Einkommenssplitting“ erfolgt. Es bewirkt die sozialrechtliche Inpflichtnahme aller Mitglieder der Bedarfsgemeinschaft. Das sozialrechtliche Einkommenssplitting ist für Partnerschaften mit Niedrigverdienst außerdem die Grundlage für die fiktive Hilfebedürftigkeit Erwerbstätiger.

Der Zugang zu aktivierenden Leistungen des SGB III und zu Eingliederungsleistungen des SGB II sollte entsprechend dem geltenden Unterhaltsrecht unabhängig davon ermöglicht werden, ob wegen des Partnereinkommens Hilfebedürftigkeit im Sinne des SGB II besteht. Frauen sollen nicht faktisch als „Versorgte“ aus der aktiven Arbeitsförderung herausfallen können.

Es ist für Kinder wichtig, die Widersprüche von sozialrechtlichen und unterhaltsrechtlichen Pflichten aufzulösen. Wenn kein Unterhaltsrechtsverhältnis zum Kind besteht, widerspricht die Anrechnung nicht gesicherter finanzieller Ansprüche dem Kindesinteresse. Nicht zu begründen ist außerdem, bei einem besonderen Bedarf Kinder abhängig davon unterschiedlich zu behandeln, ob ihre Eltern im SGB II- oder im SGB XII-Bezug sind.
Die Grundsicherung für Arbeitssuchende ist außerdem nicht der richtige Ort, um das Problem ausstehender oder mangels Unterhaltsfähigkeit nicht vorhandener Barunterhaltsansprüche von Kindern zu lösen. Wenn Kinder Barunterhaltsansprüche gegen Väter nicht realisieren können, ist es eine staatliche Aufgabe, sie dabei zu unterstützen und gegebenenfalls mit ergänzenden Leistungen aus der Unterhaltsvorschusskasse einzutreten, die zu einem Drittel vom Bund und im Übrigen von den Ländern finanziert wird. ${ }^{21}$ Dieser Weg könnte dazu beitragen, dass Kinder nicht wegen offener Barunterhaltsansprüche hilfebedürftig im Sinne des SGB II werden und neue Partner für offene finanzielle Unterhaltsansprüche anderer eintreten müssen.

21 Die Möglichkeit nach $\$ 48$ SGB I, bei Verletzung der Unterhaltspflicht das Kindergeld an die Kinder auszuzahlen, würde das Problem nur ansatzweise und bei fehlenden Unterhaltsansprüchen mangels Unterhaltsfähigkeit des zur Leistung von Barunterhalt Verpflichteten überhaupt nicht lösen können.

\section{Die Qual der Wahl - der neue Kinderzuschlag nach § 6a BKKG}

Prof. Dr. Susanne Dern

Mitglied der Kommission Recht der sozialen Sicherung, Familienlastenausgleich des djb; Hochschule Esslingen
Zum 1. Oktober 2008 erfolgte eine Novellierung des Kinderzuschlages nach $\mathbb{\$} 6$ a BKGG, die auf den ersten Blick einen begrüßenswerten Zuwachs an Autonomie insbesondere für Alleinerziehende im SGB II-Bezug beinhaltet. Auf den zweiten Blick verbergen sich in ihr jedoch gerade für diese Gruppe, die zu 96 Prozent aus Frauen besteht, kaum abschätzbare Risiken. Die Möglichkeit, vom SGB IIBezug in den Kinderzuschlag (KiZ) zu wechseln, wenn dadurch Hilfebedürftigkeit nach dem SGB II vermieden wird, ist per se nicht neu. Der Kinderzuschlag soll Familien mit nicht bedarfsdeckenden Einkommen erreichen, die sich scheuen, SGB IIAnträge zu stellen. Der Kinderzuschlag von maximal 140 Euro pro Kind ${ }^{1}$ wird gewährt, wenn die Summe aus Kinderzuschlag und Wohngeld die Leistungen zum Lebensunterhalt inklusive der Kosten der Unterkunft $(\mathbb{S} \mathbb{S} 19,22$ SGB II) übersteigt und somit Hilfebedürftigkeit i.S.d. SGB II nicht mehr besteht. Im Herbst 2008 wurde nun neben klarstellenden Korrekturen ${ }^{2}$ auch ein sogenanntes „kleines Wahlrecht“ in $\$$ 6a Abs. 1 Nr. 4 BKKG eingefügt, das es den Berechtigten „ermöglicht“, auf Leistungen nach SGB II zu verzichten und den Kinderzuschlag zu wählen, wenn ihre Hilfebedürftigkeit nur unter Außerachtlassung von Mehrbe- darfen nach $\$ 21$ SGB II vermieden wird. Mit dieser fiktiven Annahme der fehlenden Hilfebedürftigkeit haben nun unter anderem Alleinerziehende ${ }^{3}$ die Wahl, sich gegen (höhere) ALG II-Leistungen zu entscheiden, um mit dem Kinderzuschlag das fordernde, mit Sanktionsdrohungen verbundene Grundsicherungssystem zu verlassen. Dieser vermeintliche Zuwachs an Autonomie führt jedoch nicht nur zu aktuellen finanziellen Einbußen, sondern kann auch langfristig schwerwiegende, kaum mehr korrigierbare Nachteile zur Folge haben.

Zur jüngsten Geschichte des KiZ ist hier anzumerken, dass dieses Wahlrecht zunächst seitens der Arbeitsgemeinschaften als Grundsicherungsträger ignoriert bzw. konterkariert wurde. Diese sahen sich durch die Gesetzänderung veranlasst, laufende SGB II-Leistungen unter Verweis auf die zukünftig mögliche Beantragung des vermeintlich vorrangigen KiZ einzustellen, wodurch sie der Entscheidung der Betroffenen faktisch vorgriffen und diese

1 Unter 25 Jahre.

2 Zudem wurden klare Mindesteinkommensgrenzen (600 Euro für Alleinerziehende, 900 Euro für Partner) eingeführt und die Abschmelzungen für Einkommen aus Erwerbstätigkeit abgesenkt.

3 Behinderungsbedingter Mehrbedarf ( $\$ 21$ Abs. 4 SGB II) wird ebenfalls erfasst. 